\title{
Avaliação dos efeitos atmosféricos no albedo e NDVI obtidos com imagens de satélite
}

\author{
Marcus V. C. Bezerra ${ }^{1}$, Bernardo B. da Silva ${ }^{1} \&$ Bergson G . Bezerra $^{1}$
}

\section{RESUMO}

0 albedo é um elemento importante em estudos relacionados aos balanços de radiação e energia. Por conseguinte, alterações ocorridas em diversos biomas trazem grandes implicações ao conforto humano e animal. N este sentido, o monitoramento do albedo através de imagens orbitais possibilita identificar a antropização de grandes áreas e seus impactos ambientais. 0 presente trabalho foi desenvolvido com 0 objetivo de avaliar o impacto da correção atmosférica na estimativa do albedo da superfície e do NDVI, utilizando-se a metodologia tradicional do SEBAL (Bastiaanssen, 2000) e a correção atmosférica no cômputo da refletância, banda a banda (Allen et al., 2007b), para quatro imagens Landsat 5 - TM da região do Cariri cearense. 0 s resultados mostraram diminuição no albedo da superfície e acréscimo no ND VI, corrigidos os efeitos atmosféricos para todas as áreas amostrais. De acordo com o teste de Student as diferenças entre os albedos e NDVIs, obtidas segundo os dois procedimentos considerados, são significativas a nível de significância de 0,01.

Palavras chave: correção atmosférica, albedo, NDVI, Landsat 5 - TM

\section{Evaluation of the atmospheric effects on albedo and NDVI using satellite images}

\begin{abstract}
The albedo is an important element in studies related to radiation and energy balances. Consequently, changes in different biomes bring major implications for human and animal comfort. Accordingly, the monitoring of the albedo by orbital images enables to identify the anthropic influence of large areas and their environmental impact. This work was developed with the objective of assessing the impact of atmospheric correction at surface albedo and the NDVI using the traditional method of SEBAL (Bastiaanssen, 2000) and atmospheric correction band to band (Allen et al., 2007b) for four Landsat 5 TM images covering Cariri mesoregion of Ceará State. The results showed decrease in the surface albedo and increase in NDVI, corrected for atmospheric effects for all sample areas. According with Student test there was significant difference betw een the albedo and NDVI under the two methods, significant at 0.01 level.
\end{abstract}

Key words: atmospheric correction, albedo, NDVI, Landsat 5 - TM 


\section{INTRODUÇÃO}

Nos últimos anos, o sensoriamento remoto orbital tem-se tornado uma ferramenta importante no monitoramento de fenômenos meteorológicos e ambientais, pois proporciona melhor avaliação, manejo, gerenciamento e gestão dos recursos naturais, como água, solo e vegetação.

Com o propósito de se estudar a evapotranspiração - ET via sensoriamento remoto orbital, faz-se necessário investigar a influência da atmosfera na atenuação do sinal da radiação solar refletida pela superfície que é detectada pelo sensor do satélite. Tal atenuação incorpora erros na estimativa da refletância superficial que, por sua vez, influenciam as estimativas do albedo superficial $-\alpha$, parâmetro de suma importância na determinação do saldo de radiação - Rn, e de índices de vegetação, em especial o Índice de Vegetação da Diferença Normalizada - NDVI, que é um indicador da quantidade e da condição da vegetação verde.

O albedo é definido como a razão entre os fluxos de radiação solar refletido e incidente, e constitui um parâmetro importante no estudo de mudanças climáticas, desertificação, queimadas e impactos ambientais (Silva et al., 2005a). O NDVI, por sua vez, tem se tornado uma ferramenta de grande utilidade na caracterização da vegetação e de culturas agrícolas (Moreira \& Shimabukuro, 2004).

Existem diferentes algoritmos destinados ao cômputo do albedo superficial, a partir de medições realizadas nas bandas espectrais dos canais 1 e 2 do AVHRR - NOAA. Um dos mais conhecidos foi proposto por Wydick et al. (1987), o qual relaciona as refletâncias espectrais do AVHRR, medidas no topo da atmosfera, com o albedo superficial; um outro modelo muito utilizado é o proposto por Hucek \& Jacobowitz (1995). Liang (2002), por sua vez, obteve para vários sistemas (AVHRR, MODIS, ETM+ e outros), equações de transformação da refletância de diferentes bandas espectrais em albedo da superfície. Todas as parametrizações apresentadas se aplicam às refletâncias espectrais a superfície, quando feitas as devidas correções atmosféricas. Song \& Gao (1999) também propuseram um modelo de conversão das refletâncias espectrais do AVHRR em albedo superficial, com resultados muito consistentes: este modelo se baseou em medições do albedo da superfície e refletâncias espectrais medidas no topo da atmosfera, corrigidas com o modelo de transferência radiativa LOWTRAN - 7 .

Para o Landsat 5 - TM e Landsat 7 - ETM+, utilizados intensivamente na Bacia do Bear River, nos Estados Unidos (Allen et al., 2002; 2005; 2007a, b) e em diferentes áreas irrigadas do globo (Bastiaanssen, 2000) a obtenção do albedo é baseada na combinação linear da refletância das bandas 1 a 5 e 7, e em correções atmosféricas simples e que têm produzido resultados muito consistentes, dispensando a utilização de códigos de transferência radiativa de maior complexidade.

A refletância obtida pelo sensor do satélite é a mistura do sinal da atmosfera e da superfície, denominada refletância planetária. Isto ocorre devido o sensor detectar não apenas a radiação solar refletida pela superfície, mas também a radiação espalhada ou refletida pela atmosfera (Tasumi et al., 2005), fato que caracteriza a necessidade da utilização de códigos de transferência radiativa, tais como MODTRAN, LOWTRAN e
6S, além de dados de radiossondagem da área de interesse no instante e data da imagem utilizada para se fazer correções atmosféricas. A necessidade de tais dados compromete a operacionalidade do método, pois para cada imagem estudada acarretaria na utilização de campanhas de radiossondagem no local e instante da passagem do satélite. Visando evitar tais problemas, Allen et al. (2007b) desenvolveram um método prático que, baseado em dados extraídos de análises com o MODTRAN e em Modelo de Elevação Digital (MED), favorece a operacionalidade do uso da correção atmosférica banda a banda, com os sensores TM - Landsat 5 e MODIS - Terra/ Aqua.

Vários outros trabalhos têm sido desenvolvidos com o propósito de inferir correções atmosféricas, como o de Antunes et al. (2003) que fizeram correções atmosféricas em imagens TM - Landsat 5 e ETM+-Landsat 7, utilizando o modelo 6S RTM e obtiveram, para áreas vegetadas, diminuição da refletância corrigida para as bandas 1, 2 e 3 e aumento na mesma para as bandas 4, 5 e 7; já Tasumi et al. (2005), utilizaram imagens TM - Landsat 5 e observaram diferenças de cerca de $25 \%$ entre o albedo determinado utilizando o código de transferência radiativa MODTRAN e o albedo determinado pela metodologia tradicional do SEBAL. Weligepolage (2005), por sua vez, utilizou os algoritmos SEBALe S-SEBI com o sensor ASTER e concluiu que os valores do albedo e do NDVI estimados através da refletância com correção atmosférica caracterizam melhor os alvos avaliados.

Di Pace et al. (2008) observaram que a correção topográfica aplicada a imagens TM - Landsat 5 da região do submédio São Francisco, ocasionaram variações significativas na obtenção da emissividade, NDVI, albedo, temperatura da superfície e saldo de radiação. Também utilizando imagens TM - Landsat 5, Giongo et al. (2010) observaram que os valores estimados do albedo da superfície corrigidos atmosfericamente para áreas de cerrado e cana-de-açúcar, na região de Santa Rita do Passa Quatro, SP, foram consistentes aos valores observados.

Desta forma, o presente estudo tem como objetivo avaliar a influência da correção atmosférica no $\alpha$ e no NDVI, considerando a proposta do SEBAL, segundo Bastiaanssen (2000) e a correção banda a banda, proposta por Allen et al. (2007b).

\section{Material e MÉTODOS}

Utilizaram-se quatro imagens Landsat 5 - TM, da órbita/ ponto 217/065 dos dias 29 de setembro, 15 e 31 de outubro e 16 de novembro do ano de 2005. A cena estudada abrange uma área de 7.101,7 km² entre a divisa dos estados de Pernambuco e Ceará, no Nordeste brasileiro, a qual está apresentada na Figura 1, que é uma composição RGB das bandas 5, 4 e 3, ainda na Figura 1, são apresentadas as áreas amostrais denotadas por VNSA (Vegetação Nativa do Semiárido), FNA (Floresta Nacional do Araripe), AI (Área Irrigada) e FNAA (Floresta Nacional do Araripe Antropizada), onde foram feitas análises do albedo e do NDVI obtidos a partir das refletâncias sem correção e corrigidas atmosfericamente. 


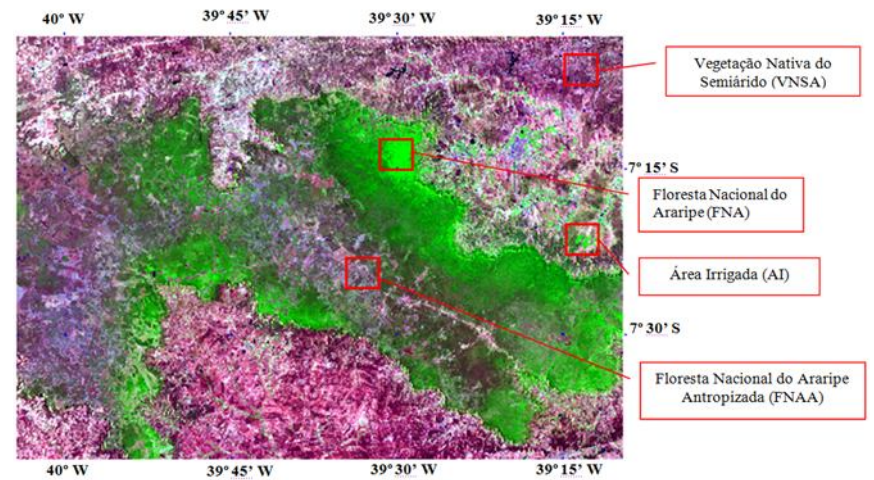

Figura 1. Região de estudo na composição RGB do Landsat 5-TM

A referida cena apresenta cobertura vegetativa bastante heterogênea composta pela Floresta Nacional do Araripe (FNA), áreas de cultivos agrícolas irrigados, os centros urbanos de Barbalha, Crato e Juazeiro do Norte, localizados no estado do Ceará, e vegetação nativa do semiárido (caatinga) de densidade variada.

A Floresta Nacional do Araripe fica localizada sobre o planalto da Chapada do Araripe, com altitude que varia de 700 a $950 \mathrm{~m}$, é composta por vegetação de alta densidade (grande área verde da Figura 1) e possui solos (principalmente latossolos) profundos, de fertilidade natural baixa, em geral arenoso e muito bem drenado, a ponto de haver pouca água de superfície, na qual a maior parte das nascentes corre para o norte, em direção ao interior do Ceará. A precipitação média anual é superior a $1.000 \mathrm{~mm}$ (Barbalha, CE, 1.049,2 $\mathrm{mm} \mathrm{ano}^{-1}$, e Crato, CE, 1.130,1 $\left.\mathrm{mm} \mathrm{ano}^{-1}\right)$.

O bioma característico da FNA é de Mata Atlântica, apresentando principalmente a seguinte composição da cobertura vegetativa: $11 \%$ de floresta úmida semiperenifolia, $49 \%$ de transição floresta úmida/cerrado e $11 \%$ de Floresta úmida degradada pelo fogo. Nesta área há atividade pecuária, pedreiras e retirada de madeira para lenha e carvão, que levaram a um estado de degradação de aproximadamente $50 \%$ de sua área.

O primeiro passo da obtenção do albedo e do NDVI, consiste na calibração radiométrica $-\mathrm{L}_{\mathrm{b}}\left(\mathrm{Wm}^{-2} \mathrm{sr}^{-1} \mathrm{~mm}^{-1}\right)$, dada pela equação seguinte utilizada por Silva et al. (2005a; b):

$$
\mathrm{L}_{b}=\mathrm{a}_{b}+\left(\frac{\mathrm{b}_{\mathrm{b}}-\mathrm{a}_{\mathrm{b}}}{255}\right) \mathrm{ND}
$$

em que:

$a_{b}$ e $b_{b}$ - coeficientes de calibração espectral do sensor (Chander \& Markham, 2003) estão descritas na Tabela 1,

ND - número digital

b - refere-se a cada banda espectral

O segundo passo consiste em determinar a refletância planetária $-r_{p}$ :

$$
\rho_{\mathrm{p}, \mathrm{b}}=\frac{\pi \cdot \mathrm{Lb}_{\mathrm{b}}}{\mathrm{k}_{\mathrm{b}} \cdot \cos \theta \cdot \mathrm{dr}}
$$

em que:

$\mathrm{k}_{\mathrm{b}}$ - irradiância solar espectral de cada banda no topo da atmosfera (Chander \& Markham, 2003) descrita na Tabela 1

$\cos \theta$ - cosseno do ângulo de incidência solar (Iqbal, 1985)

dr - inverso do quadrado da distância relativa Terra-Sol (Allen et al., 2002), dadas pelas Eqs. 3 e 4, respectivamente:

$$
\cos \theta=\cos (s) \cos (Z)+\operatorname{sen}(s) \operatorname{sen}(Z) \cos (\psi-\gamma)
$$

e

$$
\mathrm{dr}=1+0,033 \cos \left(\operatorname{DSA} \frac{2 \pi}{365}\right)
$$

em que:

$$
\begin{array}{ll}
\text { s } & \text { - inclinação da superfície } \\
Z & \text { - ângulo zenital solar } \\
\psi & \text { - ângulo azimutal solar } \\
\gamma & \text { - ângulo azimutal da superfície (todos em radiano) } \\
\text { DSA - dia sequencial do ano }(272,288,304 \text { e } 320) .
\end{array}
$$

\begin{tabular}{|c|c|c|c|c|c|}
\hline \multirow{2}{*}{ Banda } & $a_{b}$ & $b_{b}$ & \multirow{2}{*}{$\begin{array}{c}k_{b} \\
W m^{-2} \mu m^{-1}\end{array}$} & \multirow{2}{*}{$w_{p}$} & \multirow{2}{*}{$\mathbf{w}_{\mathrm{s}}$} \\
\hline & \multicolumn{2}{|c|}{$W m^{-2} s r^{-1} \mu m^{-1}$} & & & \\
\hline 1 & $-1,52$ & 193,0 & 1957 & 0,293 & 0,254 \\
\hline 2 & $-2,84$ & 365,0 & 1826 & 0,274 & 0,149 \\
\hline 3 & $-1,17$ & 264,0 & 1554 & 0,233 & 0,147 \\
\hline 4 & $-1,51$ & 221,0 & 1036 & 0,157 & 0,311 \\
\hline 5 & $-0,37$ & 30,2 & 215,0 & 0,033 & 0,102 \\
\hline 6 & 1,2378 & 15,303 & & - & - \\
\hline 7 & $-0,15$ & 16,5 & 80,67 & 0,011 & 0,036 \\
\hline
\end{tabular}

Tabela 1. Coeficientes de calibração do Landsat 5 - TM - $a_{b}$ e $b_{b}$, irradiância solar espectral de cada banda no topo da atmosfera - $k_{b}$, coeficientes peso da energia solar potencial de cada banda no topo da atmosfera - $w_{p}$ e a superfície - $w_{s}$

A refletância monocromática, corrigidos os efeitos atmosféricos ou refletância superficial banda a banda $-r_{\text {sup,b }}$, é dada por (Allen et al., 2007b):

$$
\rho_{\text {sup }, \mathrm{b}}=\frac{\rho_{\mathrm{p}, \mathrm{b}}-\rho_{\mathrm{atm}, \mathrm{b}}}{\tau_{\mathrm{in}, \mathrm{b}} . \tau_{\mathrm{asc}, \mathrm{b}}}
$$

em que:

$\rho_{\text {atm,b }}$ - refletividade atmosférica no domínio de cada banda captada pelo satélite, procedente da radiação espalhada pela atmosfera (Eq. 8)

$\tau_{\text {in,b }}$ (Eq. 4) - transmissividade atmosférica relativa ao fluxo de radiação solar que atinge a superfície na banda " $b$ "

$\tau_{\text {asc,b }}$ (Eq. 5) - transmissividade atmosférica correspondente ao fluxo de radiação solar refletido por cada pixel, banda a banda, sendo calculados segundo as expressões (Allen et al., 2007a):

$$
\tau_{\text {in, },}=C_{1} \cdot \exp \left(\frac{\mathrm{C}_{2} \mathrm{P}}{\mathrm{k}_{\mathrm{t}} \cdot \cos \theta}-\frac{\mathrm{C}_{3} \cdot \mathrm{W}+\mathrm{C}_{4}}{\cos \theta}\right)+\mathrm{C}_{5}
$$




$$
\tau_{\mathrm{asc}, \mathrm{b}}=\mathrm{C}_{1} \cdot \exp \left(\frac{\mathrm{C}_{2} \mathrm{P}}{\mathrm{k}_{\mathrm{t}} \cdot \cos \eta}-\frac{\mathrm{C}_{3} \cdot \mathrm{W}+\mathrm{C}_{4}}{\cos \eta}\right)+\mathrm{C}_{5}
$$

$\mathrm{e}$

$$
\rho_{\mathrm{atm}, \mathrm{b}}=\mathrm{C}_{\mathrm{b}} .\left(1-\tau_{\mathrm{inc}, \mathrm{b}}\right)
$$

em que:

$\mathrm{C}_{1}-\mathrm{C}_{5}$ e $\mathrm{C}_{\mathrm{b}}$ - coeficientes de correção atmosférica para cada banda (Allen et al. 2007b) na Tabela 3

$\mathrm{k}_{\mathrm{t}}$ - coeficiente de turbidez (considerado igual a 1,0)

cos $\eta$ é igual a 1 para o satélite Landsat

$\mathrm{P}_{\text {atm }}$ - pressão atmosférica $(\mathrm{kPa})$

W - água precipitável (mm), dadas pelas equações 9 e 10, respectivamente:

$$
\mathrm{P}_{\mathrm{atm}}=101,3\left(\frac{\mathrm{T}_{\mathrm{ar}}-0,0065 \mathrm{z}}{\mathrm{T}_{\mathrm{ar}}}\right)^{5,26}
$$

$$
\mathrm{W}=0,14 \cdot \mathrm{e}_{\mathrm{a}} \mathrm{P}_{\mathrm{atm}}+2,1
$$

em que:

$\mathrm{T}_{\mathrm{ar}}$ - temperatura do ar $(\mathrm{K})$

$\mathrm{e}_{\mathrm{a}}$ - pressão real do vapor próximo à superfície $(\mathrm{kPa})$

z - elevação de cada pixel acima do nível do mar (m), obtida com o MED

O albedo planetário $-\alpha_{\mathrm{p}}$ é determinado pela combinação linear seguinte:

$$
\alpha_{p}=\sum_{b=1}^{\mathrm{i}} \mathrm{w}_{\mathrm{p}, \mathrm{b}} \cdot \rho_{\mathrm{p}, \mathrm{b}}
$$

Tabela 2. Ângulo zenital - Z, ângulo azimutal solar - $\psi$, inverso do quadrado da distância relativa Terra-Sol - $d_{r^{\prime}}$ temperatura do ar - $\mathrm{T}_{\mathrm{ar}}$ e pressão real do vapor - $\mathrm{e}_{\mathrm{a}}$ no momento da passagem do satélite das cenas estudadas

\begin{tabular}{ccrrr}
\hline Data & $\mathbf{2 9 / 5 e t}$ & $\mathbf{1 5 / \text { out }}$ & \multicolumn{1}{c}{$\mathbf{3 1 / \text { out }}$} & \multicolumn{1}{c}{$\mathbf{1 6 / \text { nov }}$} \\
$\mathrm{Z}$ (graus) & 27,99 & 26,260 & 26,300 & 27,750 \\
$\psi$ (graus) & 81,07 & 94,200 & 106,970 & 116,760 \\
$\mathrm{~d}_{\mathrm{r}}$ & 0,999 & 1,008 & 1,016 & 1,024 \\
$\mathrm{~T}_{\mathrm{ar}}\left({ }^{\circ} \mathrm{C}\right)$ & 27,7 & 28,600 & 29,100 & 29,700 \\
$\mathrm{e}_{\mathrm{a}}(\mathrm{kPa})$ & 2,66 & 3,510 & 2,850 & 2,880 \\
\hline
\end{tabular}

em que:

$\mathrm{w}_{\mathrm{p}, \mathrm{b}}$ - coeficiente peso da energia solar potencial de cada banda no topo da atmosfera (Allen et al., 2002), apresentado na Tabela 1 .

A correção atmosférica do albedo segundo a metodologia de Bastiaanssen (2000) $-\alpha_{B}$, é feita com base na transmissividade atmosférica, conforme a Eq. 12. A transmissividade atmosférica - $\tau_{\text {atm }}$, por sua vez, é obtida levando-se em consideração apenas a altimetria da área, de acordo com a Eq. 13.

$$
\alpha_{\mathrm{B}}=\frac{\alpha_{\mathrm{p}}-\alpha_{\mathrm{atm}}}{\tau_{\mathrm{atm}}^{2}}
$$

em que:

$\alpha_{\mathrm{atm}}-$ albedo atmosférico $(0,03)$

$$
\tau_{\mathrm{atm}}=0,75+2 \cdot 10^{-5} \cdot \mathrm{z}
$$

em que:

z - altitude local (m), obtida com o MED de cada pixel

Já a correção atmosférica do albedo, de acordo com a proposta de Allen et al. (2007b) $-\alpha_{A}$, é feita através da combinação linear seguinte uma vez que as refletâncias espectrais $-\rho_{\text {sup.b. }}$, já foram corrigidas atmosfericamente banda a banda, conforme as Eqs. de 5 a 8:

$$
\alpha_{\mathrm{A}}=\sum_{\mathrm{b}=1}^{\mathrm{i}} \mathrm{W}_{\mathrm{s}, \mathrm{b}} . \rho_{\mathrm{sup}, \mathrm{b}}
$$

Por sua vez, o NDVI foi obtido em função das refletâncias do IV - próximo ( $\rho_{\mathrm{IV}}-$ banda 4$)$ e do vermelho $\left(\rho_{\mathrm{V}}-\right.$ banda 3$)$, sendo determinados sem correção atmosférica por $\mathrm{NDVI}_{\mathrm{SC}} \mathrm{e}$ com correção atmosférica banda a banda por $\mathrm{NDVI}_{\mathrm{CC}}$ :

$$
\mathrm{NDVI}_{\mathrm{sc}}=\frac{\rho_{\mathrm{vv}}-\rho_{\mathrm{v}}}{\rho_{\mathrm{vv}}+\rho_{\mathrm{v}}}
$$

e

$$
\mathrm{NDVI}_{\mathrm{CC}}=\frac{\rho_{\mathrm{IV} \_\mathrm{COR}}-\rho_{\mathrm{v} \_\mathrm{COR}}}{\rho_{\mathrm{IV} \_\mathrm{COR}}+\rho_{\mathrm{v} \_\mathrm{COR}}}
$$

A significância das diferenças entre os valores de $\alpha$ e NDVI, obtidos a partir das refletâncias corrigidas e não corrigidas, foi

Tabela 3. Constantes de calibração, $C_{1}-C_{5}$ e $C_{b}$ do Landsat 5 - TM

\begin{tabular}{cccccccc}
\hline Coeficiente & Banda 1 & Banda 2 & Banda 3 & Banda 4 & Banda 5 & Banda 7 \\
$C_{1}$ & 0,987 & 2,319 & 0,951 & 0,375 & 0,234 & 0,365 \\
$C_{2}$ & $-0,00071$ & $-0,00016$ & $-0,00033$ & $-0,00048$ & $-0,00101$ & $-0,00097$ \\
$C_{3}$ & 0,000036 & 0,000105 & 0,00028 & 0,005018 & 0,004336 & 0,004296 \\
$C_{4}$ & 0,0880 & 0,0437 & 0,0875 & 0,1355 & 0,0560 & 0,0155 \\
$C_{5}$ & 0,0789 & $-1,2697$ & 0,1014 & 0,6621 & 0,7757 & 0,639 \\
$C_{b}$ & 0,640 & 0,310 & 0,286 & 0,189 & 0,274 & $-0,186$ \\
\hline
\end{tabular}


avaliada através do teste de Student, aplicado à diferença de médias, a nível de significância de $1 \%$.

\section{RESULTADOS E DISCUSSÃO}

A Figura 2 apresentam os valores médios das refletâncias das bandas 1 a 5 e 7 do TM - Landsat 5, para as quatro cenas estudadas das áreas amostrais VNSA, FNA, AI e FNAA sem correção e corrigidas atmosfericamente pelo método proposto por Allen et al. (2007b), além do módulo do erro relativo entre as mesmas. Observa-se que os resultados das refletâncias das bandas 1, 2 e 3 corrigidas foram inferiores às refletâncias das mesmas bandas sem correção enquanto as refletâncias corrigidas das bandas 4, 5 e 7 foram superiores às sem correção corroborando, desta forma com os resultados obtidos por Antunes et al. (2003). Ainda segundo esses autores, a redução dos valores das refletâncias corrigidas das bandas 1 a 3 se deve ao efeito atmosférico aditivo no visível, ou seja, mais radiação é adicionada a essas bandas pela atmosfera fazendo com que a correção atmosférica cause diminuição nos valores das refletâncias das referidas bandas; já os resultados superiores das refletâncias corrigidas, em relação às sem correção, das bandas 4, 5 e 7 são atribuídas ao efeito subtrativo da atmosfera nessas bandas, principalmente à absorção pelas moléculas de água.

A banda espectral que sofreu maior impacto na correção atmosférica foi a banda 1 , com erros relativos variando de aproximadamente $93 \%$, na VNSA, a $207 \%$ na FNA, conforme pode ser observado nos gráficos da Figuras 2; observa-se ainda, que este impacto é maior em áreas de maior cobertura vegetativa, na amostra da VNSA, por exemplo, que no período da geração das imagens se encontra em estágio de dormência, ou seja, sem nenhuma folhagem em virtude do baixo teor de água no solo neste período, apresentou o menor erro relativo entre as amostras. Nas demais áreas amostrais, onde existe um teor maior de umidade, o erro foi crescente, de acordo com a densidade da cobertura vegetativa de cada área. Na amostra de FNAA, que apresenta vegetação de grande porte, porém degradada em função da adoção de práticas de manejos inadequados apresentando bastantes clareiras no seu interior, o erro percentual foi da ordem de $120 \%$, enquanto nas amostras de AI e de FNA os resultados apresentaram valores de aproximadamente 180 e $210 \%$, respectivamente. Na amostra da AI o teor de umidade do solo existente é em função do suprimento diário de água das culturas, enquanto na FNA se deve a retenção de água do solo pela vegetação densa.

Em pesquisa realizada na mesma área estudada, Bezerra et al. (2008) evidenciaram a influência do teor de umidade sobre uma parcela da FNA aplicando o SEBAL a imagens TM - Landsat 5 na determinação das densidades de fluxo de calor latente e de fluxo de calor no solo, no instante do imageamento, que, por sua vez, representou parcelas de aproximadamente 70 e $10 \%$ da densidade de saldo de radiação, respectivamente, e ET diária da ordem de 7,0 mm dia ${ }^{-1}$, já nas áreas correspondentes à VNSA, ainda no mesmo estudo, a ET foi da ordem de $3 \mathrm{~mm} \mathrm{~d}^{-1}$, enquanto nas correspondentes a AI e FNAA, a evapotranspiração foi da ordem de 6,0 e $4,0 \mathrm{~mm} \mathrm{~d}^{-1}$, respectivamente.
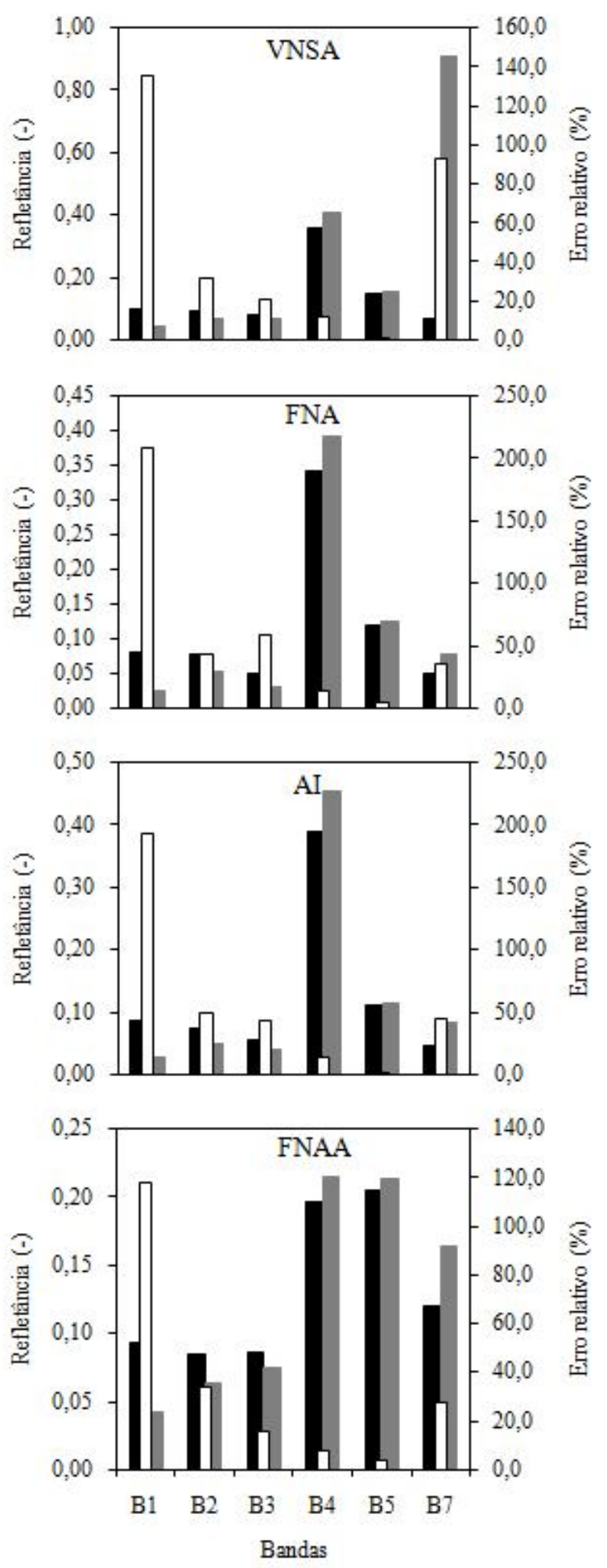

- Semcorreção nerrigida $\square$ Erro Relativo (\%)

Figura 2. Refletância média sem correção e corrigida das bandas 1 a 5 e 7 do TM - Landsat e o erro relativo nas áreas de vegetação nativa do semiárido (VNSA), Floresta $\mathrm{N}$ acional do Araripe (FN A), área irrigada (AI) e Floresta $\mathrm{N}$ acional do Araripe Antropizada (FNAA)

As bandas 3 e 4 do Landsat 5-TM têm importância relevante, pois são utilizadas nos cálculos dos principais índices de vegetação. Um índice de vegetação resulta da combinação da 
Tabela 4. Valores médios do al bedo segundo Bastiaanssen (2000) - $\alpha_{B}$ e Allen et al. (2007a) - $\alpha_{A}$ das áreas amostrais*

\begin{tabular}{|c|c|c|c|c|c|c|c|c|c|c|c|c|}
\hline \multirow[b]{2}{*}{ Data } & \multicolumn{3}{|c|}{ VNSA } & \multicolumn{3}{|c|}{ FNA } & \multicolumn{3}{|c|}{ Al } & \multicolumn{3}{|c|}{ FNAA } \\
\hline & $\alpha_{B}$ & $\alpha_{A}$ & $\underset{(\%)^{* *}}{\text { ER }}$ & $\alpha_{B}$ & $\alpha_{A}$ & $\underset{(\%)^{* *}}{\text { ER }}$ & $\alpha_{B}$ & $\alpha_{A}$ & $\underset{(\%)^{* *}}{\text { ER }}$ & $\alpha_{B}$ & $\alpha_{A}$ & $\underset{(\%)^{* *}}{\operatorname{ER}}$ \\
\hline $29 /$ set & 0,178 & 0,152 & 17,1 & 0,138 & 0,131 & 5,3 & 0,172 & 0,164 & 4,9 & 0,134 & 0,125 & 7,2 \\
\hline 15 /out & 0,177 & 0,155 & 14,2 & 0,137 & 0,130 & 5,4 & 0,167 & 0,163 & 2,5 & 0,136 & 0,127 & 7,1 \\
\hline $31 /$ out & 0,180 & 0,154 & 16,9 & 0,139 & 0,132 & 5,3 & 0,170 & 0,162 & 4,9 & 0,134 & 0,125 & 7,2 \\
\hline $16 /$ nov & 0,182 & 0,157 & 15,9 & 0,137 & 0,130 & 5,4 & 0,170 & 0,162 & 4,9 & 0,138 & 0,127 & 8,7 \\
\hline
\end{tabular}

* VNSA - vegetação nativa do semiárido; FNA - Floresta Nacional do Araripe; Al - área irrigada; FNAA - Floresta Nacional do Araripe Antropizada

** ER - erro relativo $=\left[100 \%\left(\alpha_{A}-\alpha_{B}\right) / \alpha_{A}\right]$

medida da radiação eletromagnética refletida pela vegetação, que guarda certa relação com a quantidade e o estado da vegetação na área em que ocorreu a medida (Moreira \& Shimabukuro, 2004). Observa-se nos gráficos da Figura 2, que a refletância da banda 3, quando corrigida, apresentou-se inferior ao seu próprio valor não corrigido em todas as áreas amostrais. Ainda se verifica,_nas mesmas Figuras, que o erro relativo apresentado, a exemplo da banda 1 , é maior quanto maior for a cobertura vegetativa da área amostral, em virtude do realce dado pela correção atmosférica a atividade fotossintética nas áreas de maior cobertura vegetal. $\mathrm{Na}$ área de VNSA o erro foi inferior a 10\%, passando a 15\% na FNAA, enquanto nas áreas de AI e FNA apresentou valores próximos de 40 e $60 \%$, respectivamente: já o erro relativo apresentado entre as refletâncias corrigidas e não corrigidas da banda 4, foi maior na VNSA, em torno de 20\%, enquanto nas demais áreas os erros foram da ordem de $10 \%$.

Como a refletância corrigida sofreu redução na banda 3 e acréscimo na banda 4 em relação aos resultados não-corrigidos, fica evidente que o NDVI é subestimado quando calculado com base nas refletância sem correção atmosférica, o que será discutido mais adiante, no presente estudo.

$\mathrm{Na}$ Tabela 3 está representado o albedo médio das áreas amostrais calculados com base no método de Bastiaanssen (2000) $-\alpha_{B}$ e corrigidos atmosfericamente banda a banda $-\alpha_{A}$, de acordo com Allen et al. (2007b), assim como o erro relativo entre os mesmos para cada dia de estudo. Observa-se que todas as áreas amostrais apresentaram $\mathrm{a}_{\mathrm{A}}$ inferior ao $\mathrm{a}_{\mathrm{B}}$ com erros variando de 2,5 a 17\%. Ainda na Tabela 3 é possível observar que os menores erros se encontram nas áreas da FNA, AI e FNAA, que foram inferiores a 10\%: já na área de VNSA os erros relativos foram mais acentuados, compreendidos de 14 a 17\%. Desta forma, fica evidente que as correções atmosféricas no cálculo da refletância espectral causaram maior impacto no albedo das áreas com menor cobertura vegetativa e consequente baixo teor de umidade do solo.

Sobre a FNA, os erros relativos predominantes entre $a_{B}$ e o $a_{A}$ se situaram em torno de $5 \%$, compreendendo a grande área vermelha ilustrada nas Figuras 3A a 3D, que apresentam o erro relativo entre as médias dos albedos obtidos por ambos os métodos. No restante da cena se observa que nas áreas mais próximas a FNA os erros relativos predominantes foram na faixa de 10 a 15\% (áreas amarelas). Nessas áreas a cobertura vegetativa é constituída de resquícios da vegetação típica da FNA mesclada com vegetação nativa típica do semiárido, ou seja, corresponde a uma faixa de transição FNA/Caatinga: já nas áreas mais afastadas da FNA os erros foram superiores a 15\%, com predominância na faixa de 15 a 20\% (áreas verdes), que correspondem à vegetação nativa rala.

Nas Figuras 3A a 3D é possível observar que no interior da FNA existem áreas com erros relativos na faixa de 15 a $20 \%$, o que difere totalmente do padrão apresentado pela FNA nas bordas do planalto da Chapada do Araripe, o que se deve às áreas da FNA que sofreram acentuada ação de exploração (áreas antropizadas).

Observa-se nitidamente que o NDVI obtido a partir das refletâncias corrigidas - $\mathrm{NDVI}_{\mathrm{CC}}$, foi predominantemente maior em relação ao NDVI obtido em função das refletâncias sem correção - $\mathrm{NDVI}_{\mathrm{SC}}$, em todas as áreas amostrais, como pode ser verificado na Tabela 4, com erros relativos na faixa de $6 \mathrm{a}$ $23 \%$. Também é possível observar que os menores erros correspondem a AI, inferiores a 8\%; na FNA e FNAA esses erros são em torno de $13 \%$ e 19\%, respectivamente, enquanto que para a VNSA é em torno de $22 \%$. O acréscimo no valor dos $\mathrm{NDVI}_{\mathrm{CC}}$ em relação ao $\mathrm{NDVI}_{\mathrm{SC}}$ se deve ao fato da diminuição na refletância corrigida da banda 3 do TM - Landsat 5 e ao aumento na refletância corrigida da banda 4 do referido sensor, como observado na Figura 2.

Nas Figuras 4A a 4D se encontram os mapas dos erros relativos entre os $\mathrm{NDVI}_{\mathrm{SC}}$ e o $\mathrm{NDVI}_{\mathrm{CC}}$. Nas referidas figuras se observa que sobre a FNA os erros estão na faixa de 10 a $20 \%$ (grande área amarela/laranja). Ainda sobre a FNA se nota que nas bordas do planalto da Chapada do Araripe os erros relativos entre os NDVIs foram na faixa de 10 a $15 \%$ (área laranja), menor que o valor predominante da FNA. Nas áreas próximas a FNA, que correspondem a áreas de transição, os erros estão compreendidas entre $20 \%$ e $25 \%$, e nas áreas mais afastadas essas diferenças são superiores a $25 \%$; na região interna a FNA, correspondente a áreas antropizadas os erros entre o

Tabela 5. Valores médios do NDVI sem correção - NDVI ${ }_{S C}$ e corrigido - NDVI ${ }_{C C}$ das áreas amostrais*

\begin{tabular}{|c|c|c|c|c|c|c|c|c|c|c|c|c|}
\hline \multirow{2}{*}{ Data } & \multicolumn{3}{|c|}{ VNSA } & \multicolumn{3}{|c|}{ FNA } & \multicolumn{3}{|c|}{ Al } & \multicolumn{3}{|c|}{ FNAA } \\
\hline & $\mathrm{NDVI}_{\mathrm{SC}}$ & $\mathrm{NDVI}_{\mathrm{CC}}$ & $\operatorname{ER}(\%)^{* *}$ & $\mathrm{NDVI}_{\mathrm{SC}}$ & $\mathrm{NDVI}_{\mathrm{CC}}$ & $\operatorname{ER}(\%)^{* *}$ & $\mathrm{NDVI}_{\mathrm{SC}}$ & $\mathrm{NDVI}_{\mathrm{CC}}$ & $\operatorname{ER}(\%)^{* *}$ & $\mathrm{NDVI}_{\mathrm{SC}}$ & $\mathrm{NDVI}_{\mathrm{CC}}$ & $\operatorname{ER}(\%)^{* *}$ \\
\hline $29 /$ set & 0,250 & 0,316 & 20,9 & 0,726 & 0,831 & 12,6 & 0,774 & 0,825 & 6,2 & 0,417 & 0,517 & 19,3 \\
\hline $15 /$ out & 0,246 & 0,318 & 22,6 & 0,727 & 0,836 & 13,0 & 0,769 & 0,818 & 6,0 & 0,415 & 0,511 & 18,8 \\
\hline $31 /$ out & 0,233 & 0,297 & 21,5 & 0,737 & 0,845 & 12,8 & 0,756 & 0,816 & 7,4 & 0,416 & 0,515 & 19,2 \\
\hline $16 /$ nov & 0,231 & 0,298 & 22,5 & 0,735 & 0,843 & 12,8 & 0,759 & 0,817 & 7,1 & 0,413 & 0,513 & 19,5 \\
\hline
\end{tabular}

* VNSA - vegetação nativa do semiárido; FNA - Floresta Nacional do Araripe; Al - área irrigada; FNAA - Floresta Nacional do Araripe Antropizada

** ER - erro relativo $=\left[100 \%\left(\mathrm{IVDN}_{\mathrm{cC}}-\mathrm{IVDN}_{\mathrm{SC}}\right) / \mathrm{IVDN}_{\mathrm{cC}}\right]$ 
A.

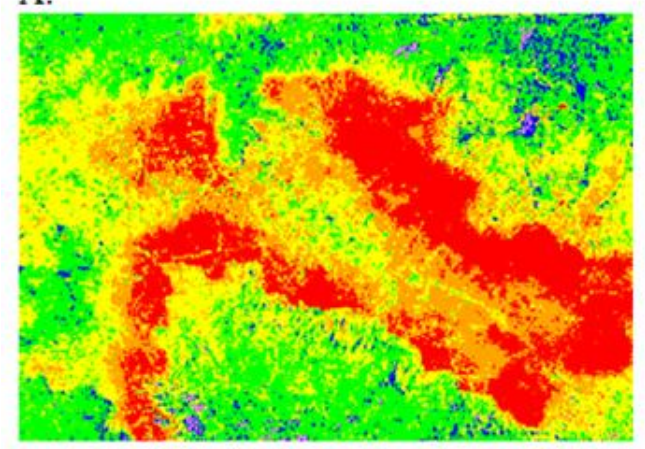

C.

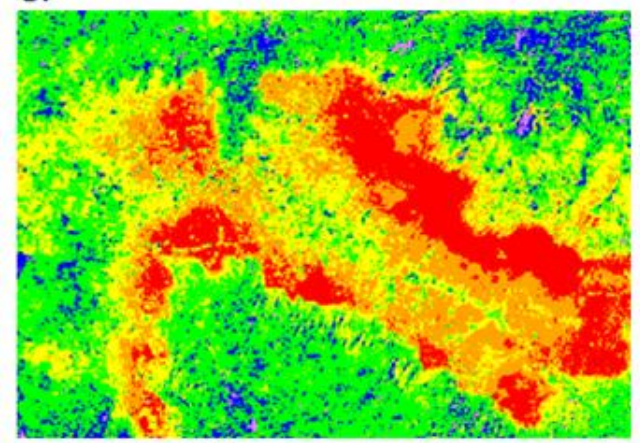

B.

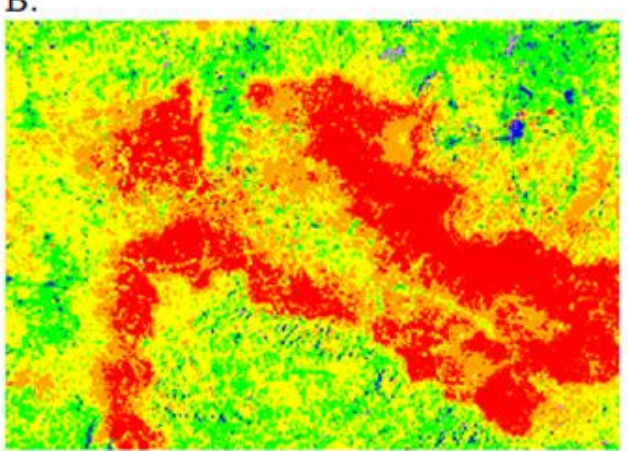

D.

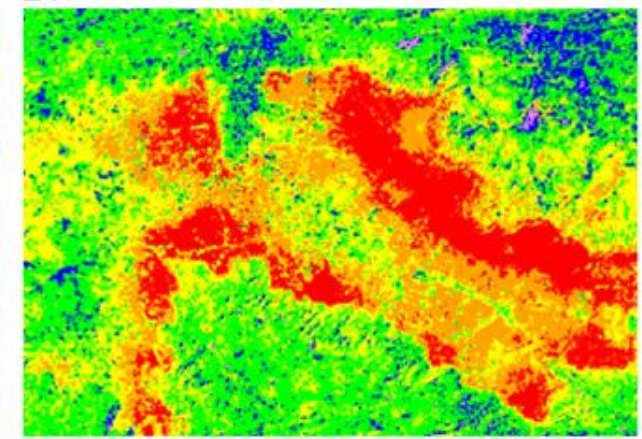

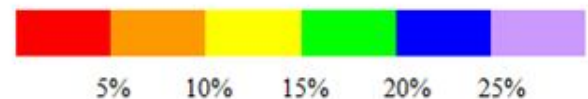

Figura 3. Mapas do erro relativo entre $0 \alpha_{B}$ e $\alpha_{A}$ em (A) 29/set/2005, (B) 15/out/2005, (C) 31/out/2005 e (D) 16/nov/2005

A.

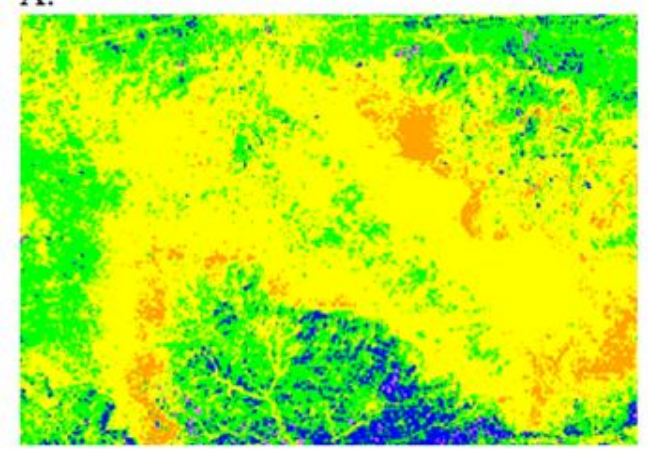

C.

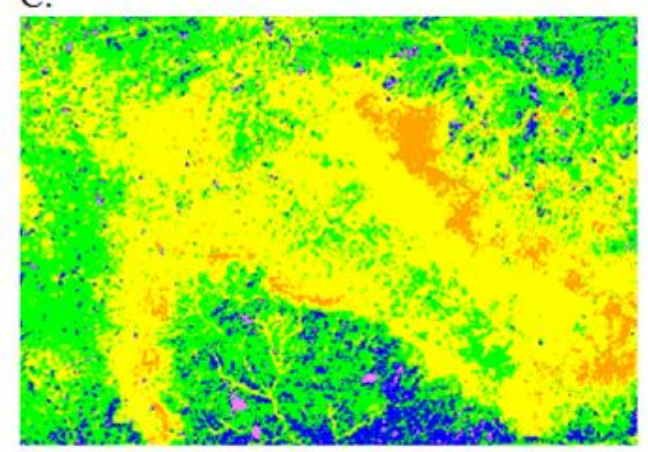

B.

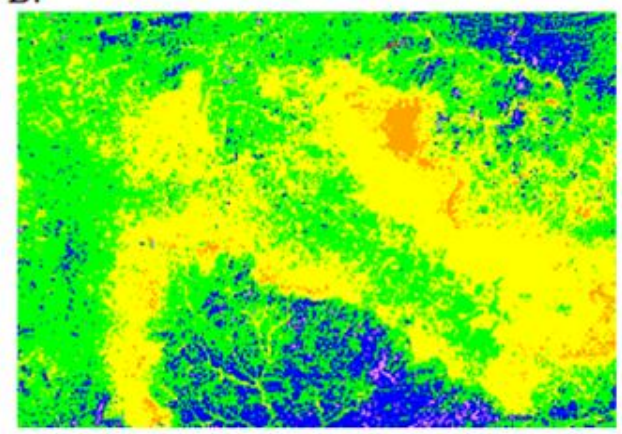

D.

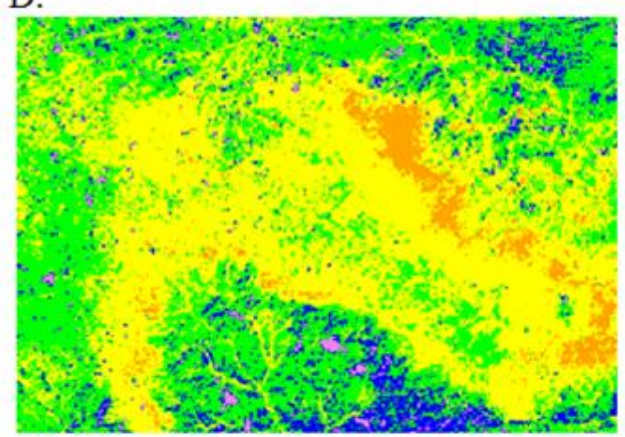

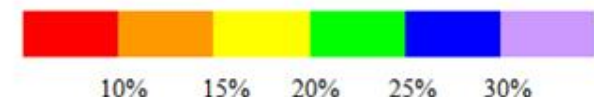

Figura 4. Mapas do erro relativo entre o N DVI e N DVI $_{c c}$ em (A) 29/set/2005, (B) 15/out/2005, (C) 31/out/2005 e (D) 16/ nov/2005 
Tabela 6. Desvio padrão para $O_{B}$ e $O_{A}$ das áreas amostrais*

\begin{tabular}{|c|c|c|c|c|c|c|c|c|}
\hline \multirow{2}{*}{ Data } & \multicolumn{2}{|c|}{ VNSA } & \multicolumn{2}{|c|}{ FNA } & \multicolumn{2}{|c|}{ Al } & \multicolumn{2}{|c|}{ FNAA } \\
\hline & $\alpha_{B}$ & $\alpha_{A}$ & $\alpha_{B}$ & $\alpha_{A}$ & $\alpha_{B}$ & $\alpha_{A}$ & $\alpha_{B}$ & $\alpha_{A}$ \\
\hline $29 /$ set & 0,024 & 0,020 & 0,005 & 0,005 & 0,009 & 0,007 & 0,021 & 0,017 \\
\hline $15 /$ out & 0,021 & 0,018 & 0,006 & 0,006 & 0,008 & 0,007 & 0,025 & 0,019 \\
\hline $31 /$ out & 0,022 & 0,018 & 0,006 & 0,007 & 0,007 & 0,008 & 0,021 & 0,017 \\
\hline $16 /$ nov & 0,023 & 0,018 & 0,006 & 0,007 & 0,008 & 0,010 & 0,024 & 0,018 \\
\hline
\end{tabular}

Tabela 7. Desvio padrão para o N DVI $\mathrm{SC}_{\mathrm{SC}}$ o NDVI $\mathrm{NC}_{\mathrm{C}}$ das áreas amostrias*

\begin{tabular}{|c|c|c|c|c|c|c|c|c|}
\hline \multirow{2}{*}{ Data } & \multicolumn{2}{|c|}{ VNSA } & \multicolumn{2}{|c|}{ FNA } & \multicolumn{2}{|c|}{ Al } & \multicolumn{2}{|c|}{ FNAA } \\
\hline & $\mathrm{NDVI}_{\mathrm{sc}}$ & $\mathrm{NDVI}_{\mathrm{CC}}$ & $\mathrm{NDVI}_{\mathrm{sc}}$ & $\mathrm{NDVI}_{\mathrm{CC}}$ & $\mathrm{NDVI}_{\mathrm{SC}}$ & $\mathrm{NDVI}_{\mathrm{CC}}$ & $\mathrm{NDVI}_{\mathrm{SC}}$ & $\mathrm{NDVI}_{\mathrm{CC}}$ \\
\hline $29 /$ set & 0,015 & 0,016 & 0,021 & 0,021 & 0,005 & 0,006 & 0,009 & 0,011 \\
\hline $15 /$ out & 0,016 & 0,018 & 0,021 & 0,020 & 0,007 & 0,008 & 0,010 & 0,013 \\
\hline $31 /$ out & 0,019 & 0,023 & 0,019 & 0,019 & 0,008 & 0,009 & 0,008 & 0,009 \\
\hline $16 /$ nov & 0,021 & 0,027 & 0,019 & 0,019 & 0,006 & 0,007 & 0,010 & 0,012 \\
\hline
\end{tabular}

"VNSA - vegetação nativa do semiárido; FNA - Floresta Nacional do Araripe; Al - área irrigada; FNAA - Floresta Nacional do Araripe Antropizada

Tabela 8. Valores do $t_{\text {calc }}$ do teste de Student para cada área amostral*

\begin{tabular}{|c|c|c|c|c|c|c|c|c|}
\hline \multirow{2}{*}{ Data } & \multicolumn{2}{|c|}{ VNSA } & \multicolumn{2}{|c|}{ FNA } & \multicolumn{2}{|c|}{$\mathrm{Al}$} & \multicolumn{2}{|c|}{ FNAA } \\
\hline & $\alpha$ & NDVI & $\alpha$ & NDVI & $\alpha$ & NDVI & $\alpha$ & NDVI \\
\hline $29 /$ set & 8,7 & $-31,4$ & 10,3 & $-36,9$ & 7,3 & $-68,2$ & 3,5 & $-73,5$ \\
\hline $15 /$ out & 8,3 & $-31,2$ & 8,6 & $-39,2$ & 3,9 & $-48,1$ & 3,0 & $-61,1$ \\
\hline $31 /$ out & 9,5 & $-22,4$ & 7,9 & $-42,0$ & 7,9 & $-52,0$ & 3,5 & $-85,8$ \\
\hline $16 /$ nov & 8,9 & $-20,5$ & 7,9 & $-42,0$ & 6,5 & $-65,7$ & 3,8 & $-66,8$ \\
\hline
\end{tabular}

"VNSA - vegetação nativa do semiárido; FNA - Floresta Nacional do Araripe; Al - área irrigada; FNAA - Floresta Nacional do Araripe Antropizada

NDVI corrigido e sem correção atmosférica estão na faixa de 20 a $25 \%$, contrastando com o padrão apresentado pela FNA; fica evidente, então, que os maiores erros ocorreram nas áreas de menor cobertura vegetativa e com baixo teor de umidade do solo.

Aplicou-se o teste estatístico de Student para verificar a significância das diferenças apresentadas entre as médias do $a_{B}$ e $a_{A}$ e do NDVI $_{S C}$ e NDVI $I_{C C}$ nas áreas amostrais. As amostras são compostas de 110 pixels e, e para os dias estudados, foram extraídos de cada um, a média e o desvio cujos resultados estão representados nas Tabelas 5 e 6 , tanto do albedo quanto do NDVI. O valor crítico para um grau de liberdade infinito para o nível de $1 \%$ de significância é de 2,326. Observa-se, assim, de acordo com a Tabela 7, que em todas as áreas amostrais, tanto para o albedo como para o NDVI, as diferenças foram estatisticamente significativas.

\section{ConClusÕes}

1. As refletâncias corrigidas foram inferiores às não corrigidas nas bandas $1,2 \mathrm{e} 3$, e superiores nas bandas 4,5 e 7 do TM - Landsat 5.

2. A banda espectral que sofreu maior impacto na correção atmosférica foi a banda 1, com erro relativo de 93 a 207\%; a magnitude do erro relativo nessa banda foi proporcional à cobertura vegetativa da área.

3. Os maiores impactos das correções atmosféricas das refletâncias foram causados tanto no albedo como no NDVI, nas áreas de menor cobertura vegetativa.

4. De acordo com o teste de Student, as diferenças entre $\alpha_{\text {B }}$ e $\alpha_{\mathrm{A}}$ e entre $\mathrm{NDVI}_{\mathrm{SC}}$ e NDVI $\mathrm{CC}_{\mathrm{C}}$, são estatisticamente significativas a nível de significância de $1 \%$.

\section{LITERATURA CITADA}

Allen, R. G.; Tasumi, M.; Morse, A.; Trezza, R. A Landsat-based energy balance and evapotranspiration model in Western US water rights regulation and planning. Irrigation and Drainage Systems, v.19, p.251-268, 2005.

Allen, R. G.; Tasumi, M.; Morse, A.; Trezza, R.; Wright, J. L.; Bastiaanssen, W.; Kramber, W.; Lorite, I.; Robinso, C. W. Satellite-based energy balance for mapping evapotranspiration with internalized calibration (METRIC) - Applications. Journal of Irrigation and Drainage Engineering, v.133, p.395-405, 2007a.

Allen, R. G.; Tasumi, M.; Trezza, R. SEBAL (Surface Energy Balance Algorithms for Land). Kimberly: University of Idaho, Advanced Training and Users Manual, 98p. 2002.

Allen, R.; Tasumi, M.; Trezza, R. Satellite-based energy balance for mapping evapotranspiration with internalized calibration (METRIC) - Model. Journal of Irrigation and Drainage Engineering, v.133, p.380-394, 2007b.

Antunes, M. A. H.; Freire, R. M. B.; Botelho, A. S. Correções atmosféricas de imagens de satélites utilizando o modelo 6S. In: Congresso Brasileiro de Cartografia, 21, 2003, Belo Horizonte. Anais... Belo Horizonte: SBC, 2003. CD Rom.

Bastiaanssen, W. G. M. SEBAL - Based sensible and latent heat fluxes in the irrigated Gediz basin, Turkey. Journal of Hydrology, v.229, p.87-100, 2000.

Bezerra, B. G.; Silva, B. B. da; Ferreira, N. J. Estimativa da evapotranspiração real diária utilizando-se imagens digitais TM - Landsat 5. Revista Brasileira de Meteorologia, v.23, p.305-317, 2008. 
Chander, G.; Markham, B. Revised Landsat-5 TM radiometric calibration producers and postcalibration dynamic rangers. IEEE Transactions on Geoscience and Remote Sensing, v.41, n.11, p.2674-2677, 2003.

Di Pace, F. T.; Silva, B. B.; Silva, V. P. R.; Silva, S. T. A. Mapeamento do saldo de radiação com imagens Lansat 5 e modelo de elevação digital. Revista Brasileira de Engenharia Agrícola e Ambiental, v.12, p.385-392, 2008.

Giongo, P. R.; Moura, G. B. A.; Silva, B. B.; Rocha, H. R.; Medeiros, S. R. R.; Nazareno, A. C. Albedo à superfície a partir de imagens Landsat 5 em áreas de cana-de-açúcar e cerrado. Revista Brasileira de Engenharia Agrícola e Ambiental, v. 14, p.279-287, 2010.

Hucek, R.; Jacobowitz, H. Impact of scene dependence on AVHRR albedo models. Journal of Atmospheric and Oceanic Technology, v.12, p.2674-2677, 1995.

Iqbal, M. An introduction to solar radiation. 1.ed. Toronto: Academic Press, 1983. 390p.

Liang, S. Narrowband to broadband conversation of land surface albedo_I Algoritms. Remote Sensing of Enviroment, v.76, p.213-238, 2002.

Moreira, M. A.; Shimabukuro, Y. E. Cálculo doíndice de vegetação a partir do sensor AVHRR. In. Ferreira, N. J. (Cord.). Aplicações Ambientais Brasileiras dos Satélites NOAA e TIRO-N. São Paulo: Oficina de Textos, 2004. cap. 4, p.79-101.
Silva, B. B.; Lopes, G. M.; Azevedo, P. V. de. Determinação do albedo de áreas irrigadas com base em imagens LANDSAT 5 - TM. Revista Brasileira de Agrometeorologia, v.13, p.201211, 2005a.

Silva, B. B.; Lopes, G. M.; Azevedo, P. V. Balanço de radiação em áreas irrigadas utilizando imagens LANDSAT 5 - TM. Revista Brasileira de Agrometeorologia, v.20, p.243-252, 2005b.

Song, J.; Gao, W. An improved method to derive surface albedo from narrowband AVHRR satellite data: narrowband to broadband conversion. Journal of Applied Meteorology, v.38, p.239-249, 1999.

Tasumi, M.; Allen, R. G.; Trezza, R.; Wright, J. L. Satellite-based energy balance to asses within population variance of crop coefficient curves. Journal of Irrigation and Drainage Engineering, v.131, p.94-109, 2005.

Weligeopolage, K. Estimation of spatial and temporal distribution of evapotranspiration by satellite remote sensing - A case study in Hupselse Beek, The Netherlands. Enschede: ITC, 2005. 127p. Thesis

Wydick, J.; Davies, P.; Gruber, A. Estimation of broadband planetary albedo from operational narrowband satellite measurements. Washington; NOAA Technical Report, NESDIS 27, 1987.32p. 